\title{
Dielectrophoretic trapping of single bacteria at carbon nanofiber nanoelectrode arrays
}

\section{Supporting Information}

\author{
Prabhu U Arumugam ${ }^{1,2, *}$, Hua Chen ${ }^{1,3}$, Alan M Cassell ${ }^{1,2}$, and Jun Li $^{1, *}$ \\ ${ }^{1}$ NASA Ames Research Center, ${ }^{2}$ University Affiliated Research Center of University of California - \\ Santa Cruz, ${ }^{3}$ ELORET \\ Moffett Field, CA 94035
}

parumugam@arc.nasa.gov and jli@arc.nasa.gov

Received August7, 2007

*Corresponding authors: JL (phone: 650604 6459, email: jli@arc.nasa.gov); PUA (phone: 650604 4338, email: parumugam@arc.nasa.gov). 
Movie. A pDEP movie showing the trapping of $\mathrm{E}$ coli at NEAs. The voltage applied is $3 \mathrm{~V}_{\mathrm{pp}}$ at $1 \mathrm{MHz}$. The field is initially off and then switched on and off. The flow velocity is $\sim 250 \mu \mathrm{m} / \mathrm{s}$. E. coli are suspended in distilled water at $10^{9}$ bacteria/mL. 
